\title{
Case Report \\ Laser Photobiomodulation for a Complex Patient with Severe Hydroxyurea-Induced Oral Ulcerations
}

\author{
Marco Cabras, ${ }^{1}$ Adriana Cafaro, ${ }^{1}$ Alessio Gambino, ${ }^{1}$ Roberto Broccoletti, \\ Ercole Romagnoli, ${ }^{2}$ Davide Marina, ${ }^{3}$ and Paolo G. Arduino ${ }^{1}$ \\ ${ }^{1}$ Department of Surgical Sciences, CIR-Dental School, University of Turin, Turin, Italy \\ ${ }^{2}$ Department of Surgical and Diagnostic Sciences (DISC), University of Genoa, Genoa, Italy \\ ${ }^{3}$ Private Practice, Milan, Italy \\ Correspondence should be addressed to Paolo G. Arduino; paologiacomo.arduino@unito.it
}

Received 16 May 2016; Revised 18 October 2016; Accepted 20 October 2016

Academic Editor: Pia L. Jornet

Copyright (C) 2016 Marco Cabras et al. This is an open access article distributed under the Creative Commons Attribution License, which permits unrestricted use, distribution, and reproduction in any medium, provided the original work is properly cited.

\begin{abstract}
Patients affected by polycythemia vera (PV), a myeloproliferative neoplasm characterized by an elevated red blood cell mass, are at high risk of vascular and thrombotic complications. Conventional therapeutic options aim at reducing vascular and thrombotic risk; low-dose aspirin and phlebotomy are first-line recommendations, for patients at low risk of thrombotic events, whereas cytoreductive therapy, usually hydroxyurea (HU) or interferon alpha, is recommended for high-risk patients. In the present study, we report the case of a patient with persistent oral ulcerations, possibly related to long-lasting HU treatment, firstly treated with topic and systemic corticosteroids and then more effectively with the addition of low-level laser therapy. Laser photobiomodulation has achieved pain control and has contributed to the healing of oral ulcers without any adverse effect; this has permitted a reduction in the dose of systemic corticosteroids and the suspension of the use of the topic ones, due to the long-term stability of oral health, even after the interruption of low-level laser therapy sessions.
\end{abstract}

\section{Introduction}

Patients affected by polycythemia vera (PV), a myeloproliferative neoplasm characterized by an elevated red blood cell mass, are at high risk of vascular and thrombotic complications; they also have reduced quality of life due to a substantial symptom burden that includes pruritus, fatigue, constitutional symptoms, microvascular disturbances, and bleeding. Conventional therapeutic options aim at reducing vascular and thrombotic risk; low-dose aspirin and phlebotomy are first-line recommendations, for patients at low risk of thrombotic events, whereas cytoreductive therapy, usually hydroxyurea $(\mathrm{HU})$ or interferon alpha, is recommended for highrisk patients [1].

Mucocutaneous ulcers are possible complicating adverse effects caused by HU; these lesions can appear right after the beginning of $\mathrm{HU}$ therapy or can be a later effect, with similar clinical presentation in both situations. The oral alterations are uncommon, but they could have a greater clinical impact because of severe pain and feeding or speaking impairment $[2,3]$.

Data from literature suggest that oral ulceration is the first occurring oral side effect of hydroxyurea, usually developing after a variable period of time since administration, ranging between 5 months and up to 3 years. Mucocutaneous lesions have been diagnosed in 167 of 3411 patients on hydroxyurea with Philadelphia-chromosome-negative chronic myeloproliferative neoplasms in a large Italian cohort [4]; of this subgroup, 27 patients presented oral ulcerations. Almost half of these patients needed to discontinue hydroxyurea with resolution of lesions in about one month. The remaining patients received local therapy, consisting of mouthwash with folic acid and vitamin A, and they obtained some symptomatic improvement. However, complete healing was achieved only after $\mathrm{HU}$ dose reduction or suspension in an average time frame of three months.

Other studies $[5,6]$ have reported $12.5 \%$ to $13 \%$ prevalence of mucocutaneous involvement in smaller cohorts of 40 and 


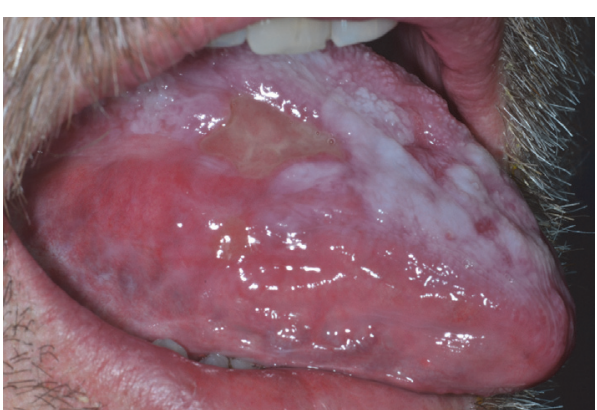

(a)

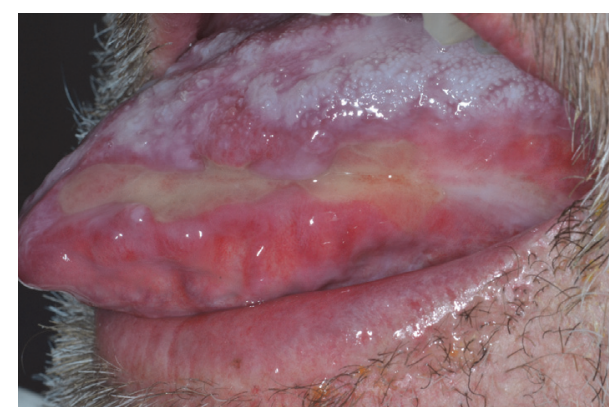

(b)

FIgURE 1: Erosive lesions presented in (a) and (b) lateral border of the tongue.

158 patients, respectively, including mucosal symptoms, such as pain and burning sensation, atrophy, and ulcers.

Actually, dose reduction or suspension of HU, if possible, can be considered as the most effective available measure [2, 4-6].

In the present study, we report the case of a patient with persistent oral ulcerations, possibly related to long-lasting HU treatment, firstly treated with topic and systemic corticosteroids and then more effectively with the addition of low-level laser therapy.

\section{Case Presentation}

A 72-year-old male (diagnosed with PV in 1994 and treated with HU since 1995) was referred to the Oral Medicine Section of the Turin University in March 2014 because of severe tongue and lips pain and difficulty in feeding and speaking. At the time being, HU dosage consisted of three $500 \mathrm{mg}$ tablets daily. At physical examination, extensive ulcerations on the tongue and lips were observed (Figure 1). Based on the clinical appearance and the topography of the lesions, a differential diagnosis between $\mathrm{HU}$ related ulcers and erosive lichen was posed. However, advice against the execution of the oral biopsy came directly from the oncologist, due to the very high level of platelets (more than $1.000 .000 / \mu \mathrm{L}$ ) detected in the patient's blood.

Due to the impossibility of solving the diagnostic dilemma through histopathological examination, we opted for an ex juvantibus approach and proceeded to administer an appropriate therapy with the intention of improving at first the patient's quality of life.

In agreement with the oncologist, a combined treatment with systemic (oral administration of $75 \mathrm{mg}$ of prednisone daily) and topic corticosteroids (clobetasol 0.05\% ointment twice daily) was established. During the following two months, a reduction of symptoms and oral lesions allowed a therapy reduction to $12.5 \mathrm{mg}$ daily of prednisone, with the topic treatment being unchanged.

After a short period of relative wellness, in July 2014 the oral situation worsened again, with diffuse extensive oral ulcerations, high levels of pain, and very poor quality of life, although HU dosage had been unaltered since our first encounter with the patient, four months before. Therapy was modified again: $75 \mathrm{mg}$ of prednisone daily was administered for two weeks and then, in accordance with the oncologist, dosage was reduced to $50 \mathrm{mg}$ daily, but with no reduction of symptoms and pain; tacrolimus ointment (Protopic ${ }^{\circledR} 0.1 \%$ ointment) was used instead of topical clobetasol, but this was to no avail.

In September 2014, the decision was taken to add photobiomodulation (low-level laser therapy, LLLT) to the pharmacological therapy, with a twice-weekly frequency. A diode laser (GaAlAs) $810 \mathrm{~nm}$ (Ora-Laser_D-Light ORALIA Medical $\mathrm{GmbH}$, Germany) was used, with spot size $1.3 \mathrm{~cm}^{2}$, power setting $100 \mathrm{~mW}$, mean power $50 \mathrm{~mW}$, duty cycle $50 \%$, frequency $10.000 \mathrm{~Hz}$, fluence $6 \mathrm{~J} / \mathrm{cm}^{2}$ (calculated as follows: Fluence $=$ power $\times$ time/surface), treatment time 160 seconds, point by point technique, not in contact (distance to the mucosa about $2 \mathrm{~mm}$ ) perpendicular to the treatment area.

Noncontact mode was specifically chosen in order to prevent the onset of additional pain which could be obtained had we preferred the direct contact between the laser probe and the oral ulcers undergoing the LLLT treatment.

After 2 weeks, a marked improvement was immediately observed with disappearance of pain and the beginning of reepithelialization of the mucosa; at that time, HU dosage consisted of three $500 \mathrm{mg}$ tablets daily interchanged with four $500 \mathrm{mg}$ tablets every other day.

However, due to hospital admission for pulmonary complications, LLLT had to be suspended for 2 months, together with a gradual reduction of prednisone dosage until complete suspension: inevitably, the interruption of these treatments caused a worsening of the oral clinical situation.

After another 4 weeks of photobiomodulation treatment sessions, the lesions had not disappeared completely, but a significant reduction of pain was observed. Due to the improvement of the oral condition, the decision was taken to stop topic corticosteroid treatment continuing with systemic treatment and laser therapy, and the remission of the lingual lesions was obtained.

After another 3 weeks, symptoms and mucosal lesions disappearance, together with functional recovery, were observed (Figure 2).

Since then, photobiomodulation treatment was concluded, together with the gradual reduction of systemic corticosteroid treatment in the following months. In the meantime, 


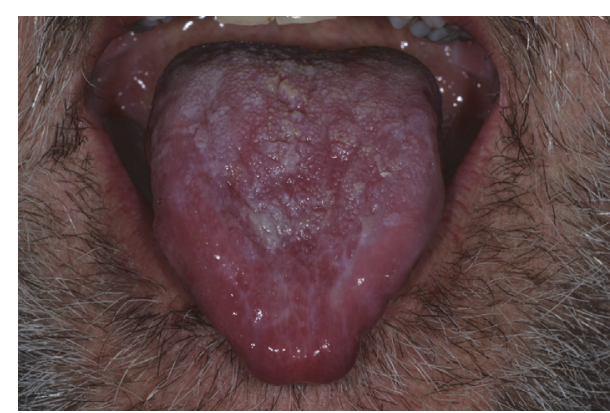

(a)

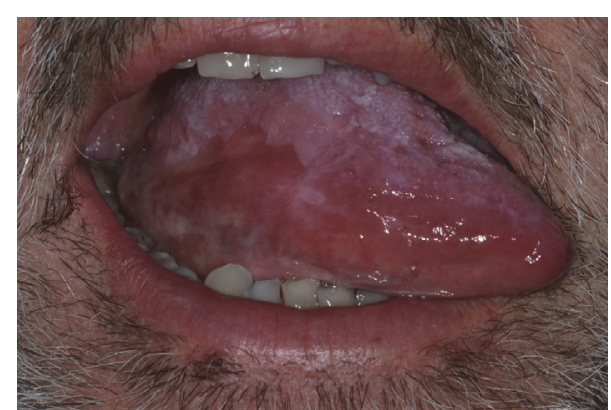

(b)

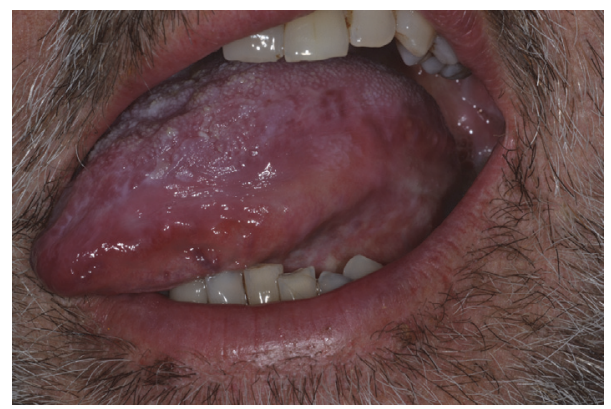

(c)

FIGURE 2: $(\mathrm{a}-\mathrm{c})$ Complete disappearance of the erosive tongue lesion at the end of the treatment provided.

HU dosage had been maintained, at the aforementioned dosage of three to four $500 \mathrm{mg}$ tablets every other day.

In September 2015, HU was finally substituted with JACAVI; since then, the patient continued to be ulcers-free; ad libitum application of topical corticosteroid was prescribed to the patient but has never been used. Our last follow-up visit, conducted in July 2016, showed no signs or symptoms at the oral cavity; meanwhile, prednisone was furthermore reduced to a minimum of $5 \mathrm{mg}$ every other day.

\section{Discussion}

PV is a classic Philadelphia-chromosome-negative myeloproliferative neoplasm (MPN), characterized primarily by an increase in red blood cell mass together with an excessive proliferation of myeloid and megakaryocytic components in the bone marrow, resulting in high red blood cells and platelet and white blood cells counts.

In the past 10 years, the pathophysiology of this condition has been defined as JAK/STAT pathway activation, almost always due to mutations in JAK2 exon 12 or 14 (JAK2 V617F) [4]. These findings have become indicative not only for diagnosis, so much that the presence of the JAK2 V617F mutation has been introduced as a major criterion in the diagnosis of PV since 2008, but also for treatment, most recently culminating in the approval of JAK inhibitors as an alternative for patients who are resistant or intolerant to $\mathrm{HU}$ [1].

Dresler and Stein described the synthesis of HU in 1869; the antitumor activity of this drug was reported in the 1960s, when it was tested against mouse leukemia and other malignancies. In the 1990s, HU was described as an inhibitor of ribonucleotide reductase, an enzyme involved in the synthesis of deoxyribonucleotides: by depleting the pool of deoxyribonucleotides, $\mathrm{HU}$ inhibits DNA synthesis, arresting the cell cycle at the G1/S phase [7].

On the other hand, skin and mucosal side effects are not completely understood and usually occur following longterm therapy; cutaneous abnormalities include xerosis, ichthyosiform lesions, dark brown pigmentation of skin folds and nails, and malleolar ulcers as well as malignant tumors [8-10].

Oral lesions are rare complications that can rapidly develop right after the start of the therapy [2] or as the side effect of a long-term exposure [3]; they often appear as painful ulcers, sometimes associated with skin lesions. Glossitis and stomatitis with intense erythema may also be observed $[6,11]$.

According to literature, there is no specific protocol for management of $\mathrm{HU}$ oral ulcers, apart from the dose reduction or suspension of $\mathrm{HU}$ itself. In the largest cohort of patients undergoing $\mathrm{HU}$, a mouthwash containing folic acid and vitamin A was prescribed, obtaining symptomatic improvement; however, no details regarding the methods (dosage, timing, etc.) are reported; moreover, the authors declared that healing was achieved only after $\mathrm{HU}$ dose reduction or suspension in an average time frame of three months [4].

To our knowledge, only two cases of oral cancer following long-term treatment with HU have been reported $[12,13]$.

Therapeutic use of laser light was introduced in 1966, when Endre Mester published the first scientific article concerning stimulating and nonthermal effects of ruby laser on mice skin [14]. 
The energy of certain wavelengths $(635-1100 \mathrm{~nm})$ is absorbed by photoacceptor chromophores in the respiratory chain (cytochrome $\mathrm{c}$ oxidase) in mitochondria: this results in a change in the redox status of the cell, together with an increase in ATP production and cell proliferation rate [1517], thanks to the activation of the nucleic acids, proteins, and enzymes regulation paths. It also participates in the regulation of the level of cytokines and growth factors and inflammation and tissue oxygenation mediators.

These biochemical cell changes lead to advantages as an increase in the speed of wound healing and a marked analgesic effect [15-18].

The rationale of using photobiomodulation is related to the possibility of accelerating the healing process of ongoing oral ulcers, as shown and reported by several studies [19-22].

In this case report laser photobiomodulation has achieved pain control and has contributed to the healing of oral ulcers without any adverse effect; this has permitted a reduction in the dose of systemic corticosteroids and the suspension of the use of the topic ones, due to the long-term stability of oral health, even after the interruption of LLLT sessions.

\section{Disclosure}

The authors alone are responsible for the content and writing of the paper.

\section{Competing Interests}

The authors report no declarations of interest.

\section{References}

[1] M. H. Griesshammer, H. Gisslinger, and R. Mesa, "Current and future treatment options for polycythemia vera," Annals of Hematology, vol. 94, no. 6, pp. 901-910, 2015.

[2] M. Badawi, S. Almazrooa, F. Azher, and F. Alsayes, "Hydroxyurea-induced oral ulceration," Oral Surgery, Oral Medicine, Oral Pathology and Oral Radiology, vol. 120, no. 6, pp. e232-e234, 2015.

[3] R. Mendonça, L. A. Gueiros, K. Capellaro, V. R. P. Pinheiro, and M. A. Lopes, "Oral lesions associated with hydroxyurea treatment," Indian Journal of Dental Research, vol. 22, no. 6, pp. 869870, 2011.

[4] E. Antonioli, P. Guglielmelli, L. Pieri et al., "Hydroxyurea-related toxicity in 3,411 patients with Ph'-negative MPN," American Journal of Hematology, vol. 87, no. 5, pp. 552-554, 2012.

[5] A. Martínez-Trillos, A. Gaya, M. Maffioli et al., "Efficacy and tolerability of hydroxyurea in the treatment of the hyperproliferative manifestations of myelofibrosis: results in 40 patients," Annals of Hematology, vol. 89, no. 12, pp. 1233-1237, 2010.

[6] C. Vassallo, F. Passamonti, S. Merante et al., "Muco-cutaneous changes during long-term therapy with hydroxyurea in chronic myeloid leukaemia," Clinical and Experimental Dermatology, vol. 26, no. 2, pp. 141-148, 2001.

[7] D. Dingli and A. Tefferi, "Hydroxyurea: the drug of choice for polycythemia vera and essential thrombocythemia," Current Hematologic Malignancy Reports, vol. 1, no. 2, pp. 69-74, 2006.

[8] N.-P. Hoff, S. Akanay-Diesel, U. Pippirs, K.-W. Schulte, and S. Hanneken, "Cutaneous side effects of hydroxyurea treatment for polycythemia vera," Hautarzt, vol. 60, no. 10, pp. 783-787, 2009.

[9] S. Boneberger, R. A. Rupec, and T. Ruzicka, "Ulcers following therapy with hydroxyurea. Three case reports and review of the literature," Hautarzt, vol. 61, no. 7, pp. 598-602, 2010.

[10] E. R. de França, M. A. G. Teixeira, K. D. F. Matias, D. E. C. M. Antunes, R. D. A. Braz, and C. E. F. Silva, "Cutaneous effects after prolongaded use of hydroxyurea in polycythemia Vera," Anais Brasileiros de Dermatologia, vol. 86, no. 4, pp. 751-754, 2011.

[11] H. Brincker and B. E. Christensen, "Acute mucocutaneous toxicity following high-dose hydroxyurea," Cancer Chemotherapy and Pharmacology, vol. 32, no. 6, pp. 496-497, 1993.

[12] E. Estève, V. Georgescu, P. Heitzmann, and L. Martin, "Multiple skin and mouth squamous cell carcinomas related to long-term treatment with hydroxyurea," Annales de Dermatologie et de Venereologie, vol. 128, no. 8-9, pp. 919-921, 2001.

[13] M. De Benedittis, M. Petruzzi, C. Giardina, L. Lo Muzio, G. Favia, and R. Serpico, "Oral squamous cell carcinoma during long-term treatment with hydroxyurea," Clinical and Experimental Dermatology, vol. 29, no. 6, pp. 605-607, 2004.

[14] E. Mester, "The use of the laser beam in therapy," Orvosi Hetilap, vol. 107, no. 22, pp. 1012-1016, 1966.

[15] M. R. Hamblin and T. N. Demidova, "Mechanisms of low level light therapy," in Proceedings of the Mechanisms for Low-Light Therapy, vol. 6140 of Proceedings of SPIE, San Jose, Calif, USA, January 2006.

[16] A. Amaroli, S. Ravera, S. Parker, I. Panfoli, A. Benedicenti, and S. Benedicenti, "The protozoan, Paramecium primaurelia, as a non-sentient model to test laser light irradiation: the effects of an $808 \mathrm{~nm}$ infrared laser diode on cellular respiration," Alternatives to Laboratory Animals, vol. 43, no. 3, pp. 155-162, 2015.

[17] S. Benedicenti, I. M. Pepe, F. Angiero, and A. Benedicenti, "Intracellular ATP level increases in lymphocytes irradiated with infrared laser light of wavelength $904 \mathrm{~nm}$," Photomedicine and Laser Surgery, vol. 26, no. 5, pp. 451-453, 2008.

[18] T. Karu, "Mitochondrial mechanisms of photobiomodulation in context of new data about multiple roles of ATP," Photomedicine and Laser Surgery, vol. 28, no. 2, pp. 159-160, 2010.

[19] D. H. Hawkins and H. Abrahamse, "The role of laser fluence in cell viability, proliferation, and membrane integrity of wounded human skin fibroblasts following Helium-Neon laser irradiation," Lasers in Surgery and Medicine, vol. 38, no. 1, pp. 74-83, 2006.

[20] J. A. D. A. C. Piva, E. M. D. C. Abreu, V. D. S. Silva, and R. A. Nicolau, "Effect of low-level laser therapy on the initial stages of tissue repair: basic principles," Anais Brasileiros de Dermatologia, vol. 86, no. 5, pp. 947-954, 2011.

[21] F. C. F. da Silva Calisto, S. L. da Silva Calisto, A. P. de Souza, C. M. França, A. P. de Lima Ferreira, and M. B. Moreira, "Use of lowpower laser to assist the healing of traumatic wounds in rats," Acta Cirurgica Brasileira, vol. 30, no. 3, pp. 204-208, 2015.

[22] D. P. Kuffler, "Photobiomodulation in promoting wound healing: a review," Regenerative Medicine, vol. 11, no. 1, pp. 107-122, 2016. 


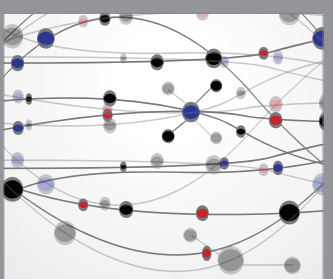

The Scientific World Journal
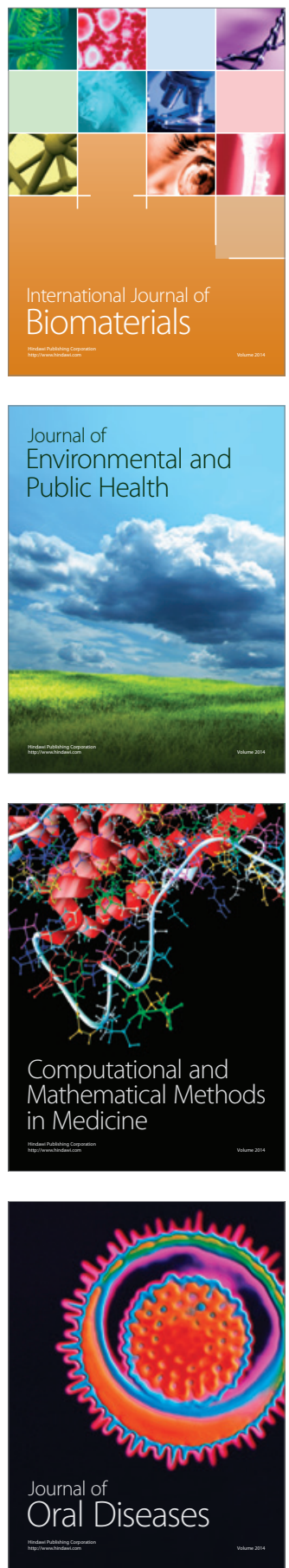
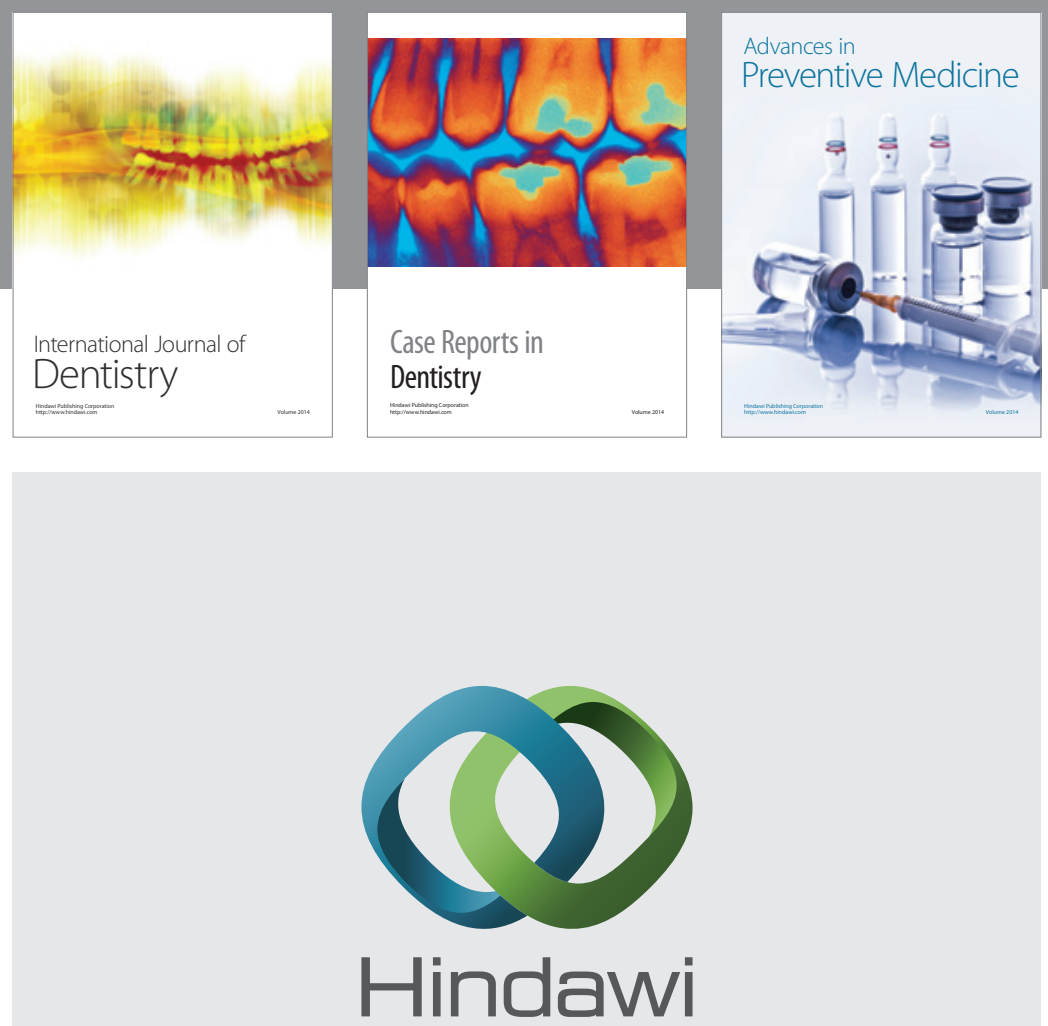

Submit your manuscripts at

http://www.hindawi.com
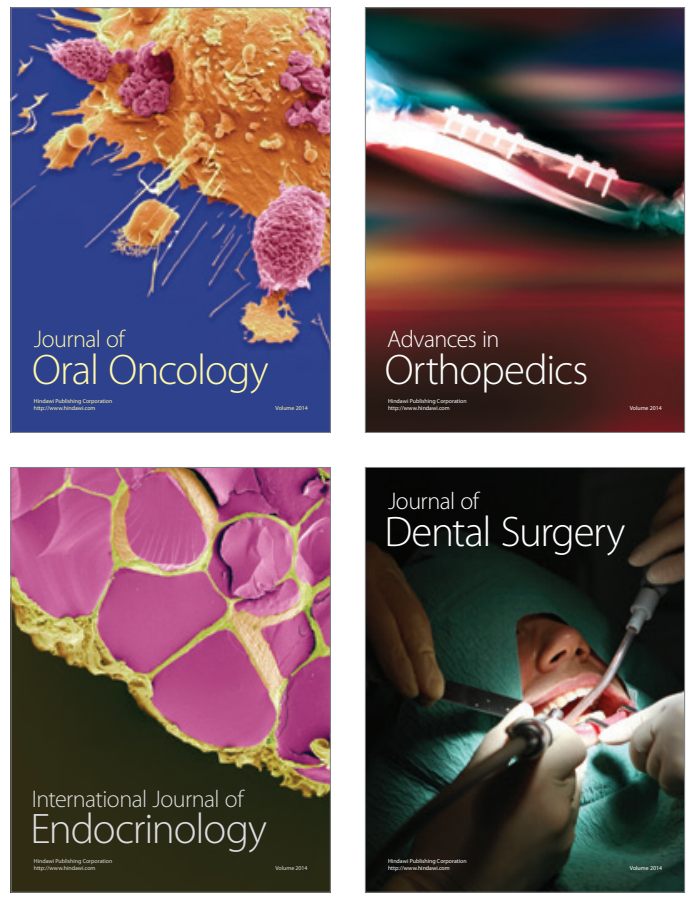
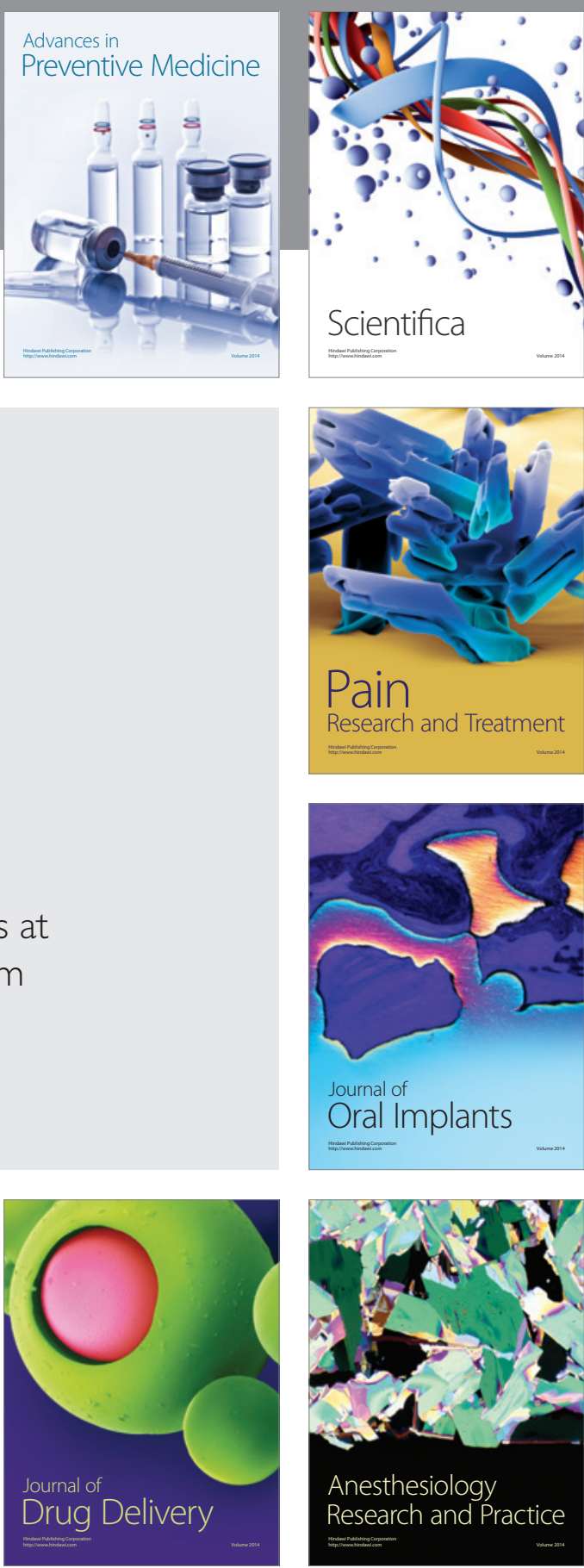

Scientifica
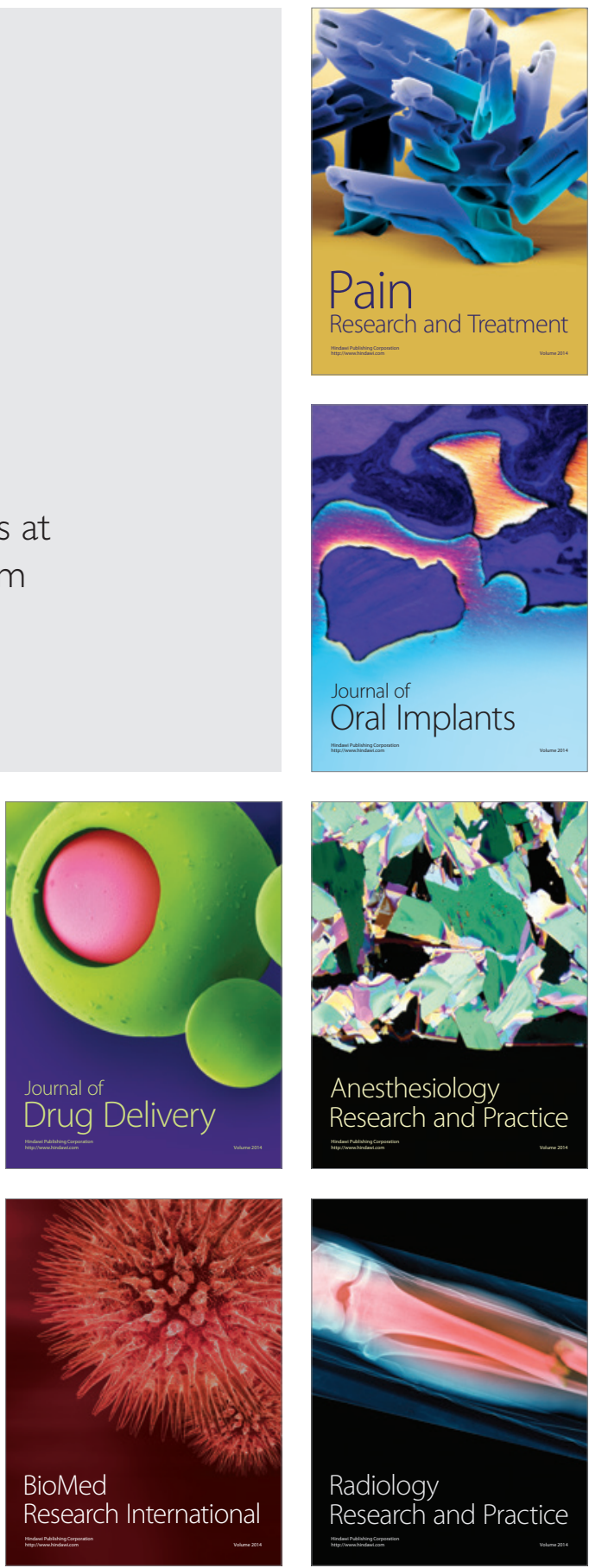